\section{DOES THE MODE OF DELIVERY INFLUENCE THE NATURAL HISTORY OF UNTREATED CERVICAL INTRAEPITHELIAL NEOPLASIA?}

S Espuelas*, E Miralpeix Rovira, L Fernandez-Sanahuja, C Roura Coll, JM Sole-Sedeno, A Salvado, J Castella, G Mancebo. Hospital del Mar, Gynecologic Oncology, Barcelona, Spain

\subsection{6/ijgc-2021-ESG0.246}

Introduction/Background* Abnormal cervical cytology has been reported in approximately 5\% of pregnancies. Dynamic cervical changes produced during labor and vaginal delivery such as dilation, epithelial desquamation and immunologic/repair processes can be associated with higher regression rates of cervical intraepithelial (CIN) lesions. However, the published literature reveals heterogeneous data and controversial results about this effect.

The objective of this study is to evaluate the influence of the mode of delivery on the histologic regression, persistence and progression rates of CIN.

Methodology A retrospective cohort study including all patients who gave birth in Hospital del Mar (Barcelona, Spain) during 2015 and 2016 was performed, identifying patients with an abnormal cervical cytology previous to delivery. Subjects were required to have postpartum follow-up that included cervical cytology between 2-9 months postpartum.

The test used was liquid-based cytology and abnormal results were classified in atypical squamous cells of undetermined significance (ASCUS), low-grade squamous intraepithelial lesion (L-SIL), high-grade squamous intraepithelial lesion (H-SIL) and atypical glandular cells (ACG).

The evolution of lesions is evaluated according to whether regression, stability or progression occurs. Rates of regression and progression of cervical lesions according to the mode of delivery (vaginal vs cesarian section) were compared. Statistical analysis was performed by Chi-square test.

Result(s)* Data from 2586 pregnant women was revised, finding 197(7.6\%) women with abnormal cytology, of which 122 women met inclusion criteria, 85(69.7\%) delivered vaginally and $37(30.3 \%)$ by cesarean section. Regression occurred in 56 (65.9\%) patients with vaginal delivery and in 28(75.7\%) with cesarean section $(\mathrm{p}=0.395)$. Progression occured in 15 cases, $8(10.5 \%)$ delivered vaginally and $7(18.9 \%)$ by cesarean section $(\mathrm{p}=0.228)$.

The global regression rate was $69.2 \%$ for H-SIL, $63.5 \%$ for L-SIL and $75 \%$ for ASCUS. None of H-SIL progressed to invasive carcinoma and only one case of ACG resulted into H-SIL in postpartum.

Conclusion* In the group of patients studied, there are no differences in the rates of regression or progression of cervical lesions depending on whether they have a vaginal delivery or cesarean section.

This study reports a high regression rates and low progression rates after delivery supporting that a conservative management of CIN during pregnancy is safe.

\section{RESULTS AFTER CONSERVATIVE SURGERY OF STAGE II/} III SEROUS BORDERLINE OVARIAN TUMORS

S Gouy* ${ }^{*}$ S Maria, A Maulard, M Faron, A Leary, P Pautier, C Chargari, C Genestie, P Morice. Gustave Roussy, Villejuif, France

10.1136/ijgc-2021-ESG0.247
Introduction/Background* The aim of this study was to assess the outcomes of a large series of patients treated conservatively for a stage II or III serous borderline tumors of the ovary (SBOTs) with a long-term follow-up.

Methodology Patients with SBOTs and peritoneal implants, treated in or referred to our institution, were retrospectively reviewed. Outcomes of patients treated conservatively (preservation of the uterus and at least a part of one ovary) to promote subsequent fertility were specifically analyzed.

Result(s)* Between 1971 and 2017, 212 patients were identified and followed-up. Among them, 65 underwent a conservative treatment. Eight patients had invasive implants. Among patients treated conservatively, 38 (58\%) patients recurred. Twenty-eight recurrences were observed under the form of borderline tumor on spared ovary and/or noninvasive implants, but 8 patients had a recurrence under the form of invasive disease. Compared to radical surgery, the use of a conservative treatment $(\mathrm{p}<.0001)$ was a prognostic factors on disease free survival (DFS), but without impact on overall survival (OS). Nevertheless, 3 deaths occurred. Twenty-four pregnancies (13 spontaneous) were observed in 20 patients (29 patients wishing to be pregnant).

Conclusion* In this series collecting the largest number of patients undergoing conservative surgery for stage II/III SBOTs, spontaneous pregnancies can be achieved after conservative treatment of advanced-stage disease, but the recurrence rate is high, and 3 deaths were observed. These patients spared their fertility but with a high rate of recurrence. Uncertainties about safety of conservative treatment should be exposed to them.

\section{FERTILITY SPARING SURGERY IN CERVICAL CANCER PATIENTS OUTSIDE CONTROLLED TRIALS - A MULTICENTER RETROSPECTIVE COHORT TRIAL (CEEGOG CX-03; ENGOT-CX14)}

1J Sláma*, ${ }^{2} S$ Kommoss, ${ }^{3} \mathrm{G}$ Scambia, ${ }^{4} \mathrm{G}$ Ferron, ${ }^{5} \mathrm{Z}$ Novák, ${ }^{6}$ I Runnebaum, ${ }^{3} \mathrm{~A}$ Fagotti, ${ }^{7} \mathrm{~F}$ Narducci, ${ }^{8} \mathrm{O}$ Matylevich, ${ }^{9} \mathrm{~J}$ Holly, ${ }^{10} \mathrm{~F}$ Raspagliesi, ${ }^{11} \mathrm{AS}$ Bats, ${ }^{12} \mathrm{~V}$ Kopetskyi, ${ }^{13} \mathrm{~A}$ El-Balat, ${ }^{3} \mathrm{G}$ Corrado, ${ }^{14} \mathrm{~F}$ Anas, ${ }^{15} \mathrm{~N}$ Volodko, ${ }^{16} \mathrm{~T}$ Piatnytska, ${ }^{1} \mathrm{~L}$ Fricová, ${ }^{1} \mathrm{D}$ Cibula. ${ }^{1}$ First Faculty of Medicine, Charles University and General University Hospital, Department of Gynecology and Obstetrics, CEEGOG, Prague, Czech Republic; '2Tüebingen University Hospital, Department of Women's Health, AGO Study Group, Tüebingen, Germany; ${ }^{3}$ Fondazione Policlinico Universitario A. Gemelli IRCCS, Catholic University of the Sacred Heart Rome Gynecologic Oncology Unit, MITO, Rome, Italy; ${ }^{4}$ Claudius Regaud Institute - University Cancer Institute, Department of Gynecological Oncology, GINECO, Toulouse, France; ${ }^{5}$ Hungarian National Institute of Oncology, Department of Gynecology, CEEGOG, Budapest, Hungary; ${ }^{6}$ Jena University Hospital, Friedrich Schiller University, Department of Gynecology and Reproductive Medicine, AGO Study Group, Jena, Germany: ${ }^{7}$ Centre Oscar Lambret, Department of Gynecological Oncology, GINECO, Lille, France; ${ }^{8}$ N.N. Alexandrov National Cancer Centre of Belarus, Gynecologic Oncology Department, CEEGOG, Minsk, Belarus; ${ }^{9}$ Evangelical Clinic Essen Mitte, Department of Gynecological Oncology, AGO Study Group, Essen, Germany; ${ }^{10}$ Fondazione IRCCS Istituto Nazionale Tumori - Milan, Department of Gynecological Oncology, MITO, Milan, Italy; ${ }^{11}$ European Georges Pompidou Hospital, Gynaecological Oncological and Breast Surgery, GINECO, Paris, France; ${ }^{12}$ National Cancer Institute, Department of Gynecologic Oncology, CEEGOG, Kyev, Ukraine; ${ }^{13}$ University Clinic Frankfurt, Goethe-University, Department of Gynecology and Obstetrics, AGO Study Group, Frankfurt am Main, Germany; ${ }^{14}$ University of Medicine and Pharmacy Târgu Mureș, First Obstetrics and Gynecology Clinic, CEEGOG, Târgu Mureş, Romania; ${ }^{15}$ Danylo Halytsky Lviv national medical university , Lviv Regional Oncological Center, CEEGOG, Lviv, Ukraine; ${ }^{16}$ Khmelnytskyi Regional Oncological Dispensary, Regional Oncological Dispensary, CEEGOG, Khmelnytskyi, Ukraine

\subsection{6/ijgc-2021-ESGO.248}

Introduction/Background* According to current guidelines fertility-sparing treatment (FST) in cervical cancer patients should follow the same principles as in patients without fertility 
preservation. The literature from the last years, however, show a trend towards less-radical procedures. Although oncological outcomes are reported to be equal or better than in non-FST, published groups are small, mostly single institutional, inclusion criteria vary, and treatment is not uniform. The aim of this study was to collect retrospective data from multiple institutions across several countries on oncological and reproductive outcomes after FST.

Methodology Included were cervical cancer patients between 18-40 years with stages $\geq$ IA1+LVSI, who underwent any type of FST and completed follow-up of at least 6 months. Patients were eligible irrespective of neoadjuvant chemotherapy, histotype or tumour grade.
Result(s)* Altogether 733 patients from 44 centers in 13 countries were eligible for analyses. Median follow-up of the whole cohort was 6 years (1.2-14.5). More than half of the cases had stage IB1 $(54.2 \%$; 397/733). Mean age of patients was 32 years and two thirds were nulliparous (484/733). Most common surgical procedure was conization (48\%). Repeated cervical surgery was performed in 161 patients (21.9\%), most frequently re-conization $(57.8 \%)$. Less than half of the patients $(49.2 \% ; 361 / 733)$ attempted to conceive after treatment. Out of them, 138 (38.2\%) got pregnant and 100 (27.7\%) successfully delivered. Pre-cancer recurrence was diagnosed in 22 (3\%) patients, 51 (7\%) patients had cancer recurrence and 19 $(2.6 \%)$ died of disease. Three times higher risk of recurrence

Abstract 991 Table 1 Characteristics of cohort

\begin{tabular}{|c|c|c|c|c|c|c|c|}
\hline & T1a1 L1 (N=208) & $\mathrm{T} 1 \mathrm{a} 2(\mathrm{~N}=102)$ & $\mathrm{T} 1 \mathrm{~b} 1 \leq 2 \mathrm{~cm}(\mathrm{~N}=356)$ & $\mathrm{T} 1 \mathrm{~b}>2 \mathrm{~cm}(\mathrm{~N}=41)$ & $\mathrm{T} 1 \mathrm{~b} 2(\mathrm{~N}=19)$ & $\mathrm{T} 2(\mathrm{~N}=7)$ & p-value \\
\hline \multicolumn{8}{|l|}{ Parity before diagnosis } \\
\hline Nullipara & $125(60.1 \%)$ & $66(64.7 \%)$ & $250(70.2 \%)$ & $24(58.5 \%)$ & $14(73.7 \%)$ & $5(71.4 \%)$ & 0.162 \\
\hline Primipara & $54(26.0 \%)$ & $27(26.5 \%)$ & $82(23.0 \%)$ & $15(36.6 \%)$ & $4(21.1 \%)$ & $2(28.6 \%)$ & \\
\hline Multipara & $29(13.9 \%)$ & $9(8.8 \%)$ & $24(6.7 \%)$ & $2(4.9 \%)$ & $1(5.3 \%)$ & $0(0.0 \%)$ & \\
\hline \multicolumn{8}{|c|}{ Age at first diagnosis of cervical cancer [years] } \\
\hline mean $(S D)$ & $33.1(4.7)$ & $33.2(4.4)$ & $31.7(4.5)$ & $30.8(3.4)$ & $29.4(5.1)$ & $33.1(5.2)$ & $<0.001$ \\
\hline median $\left(5-95^{\text {th }}\right.$ percentile) & $32.7(25.9-40.8)$ & $33.0(27.4-40.0)$ & $31.9(24.4-39.3)$ & $30.5(26.2-36.5)$ & $28.8(19.3-38.8)$ & $31.4(27.0-42.8)$ & \\
\hline \multicolumn{8}{|l|}{ Diagnostic procedure } \\
\hline Biopsy & $56(26.9 \%)$ & $23(22.5 \%)$ & $139(39.0 \%)$ & $24(58.5 \%)$ & $15(78.9 \%)$ & $5(71.4 \%)$ & $<0.001$ \\
\hline Conization & $131(63.0 \%)$ & $78(76.5 \%)$ & $212(59.6 \%)$ & $16(39.0 \%)$ & $3(15.8 \%)$ & $2(28.6 \%)$ & \\
\hline Pap smear & $14(6.7 \%)$ & $1(1.0 \%)$ & $4(1.1 \%)$ & $1(2.4 \%)$ & $0(0.0 \%)$ & $0(0.0 \%)$ & \\
\hline \multicolumn{8}{|l|}{ Histological type } \\
\hline Adenocancer & $39(18.8 \%)$ & $25(24.5 \%)$ & $101(28.4 \%)$ & $9(22.0 \%)$ & $3(15.8 \%)$ & $1(14.3 \%)$ & 0.214 \\
\hline Adenosquamous & $7(3.4 \%)$ & $4(3.9 \%)$ & $20(5.6 \%)$ & $1(2.4 \%)$ & $2(10.5 \%)$ & $0(0.0 \%)$ & \\
\hline Squamous & $161(77.4 \%)$ & $73(71.6 \%)$ & $232(65.2 \%)$ & $30(73.2 \%)$ & $13(68.4 \%)$ & $6(85.7 \%)$ & \\
\hline Other & $1(0.5 \%)$ & $0(0.0 \%)$ & $3(0.8 \%)$ & $0(0.0 \%)$ & $1(5.3 \%)$ & $0(0.0 \%)$ & \\
\hline Lymphovascular space invasion & $n=173$ & $\mathrm{n}=92$ & $\mathrm{n}=318$ & $n=41$ & $\mathrm{n}=18$ & $n=4$ & \\
\hline No & $0(0 \%)$ & $69(75.0 \%)$ & $213(67.0 \%)$ & $25(61.0 \%)$ & $7(38.9 \%)$ & $1(25.0 \%)$ & $<0.001$ \\
\hline Yes & $173(100 \%)$ & $23(25.0 \%)$ & $105(33.0 \%)$ & $16(39.0 \%)$ & $11(61.1 \%)$ & $3(75.0 \%)$ & \\
\hline \multicolumn{8}{|l|}{ Neoadjuvant chemotherapy } \\
\hline No & $207(99.5 \%)$ & $102(100.0 \%)$ & $330(92.7 \%)$ & $34(82.9 \%)$ & $11(57.9 \%)$ & $2(28.6 \%)$ & $<0.001$ \\
\hline Yes & $1(0.5 \%)$ & $0(0.0 \%)$ & $26(7.3 \%)$ & $7(17.1 \%)$ & $8(42.1 \%)$ & $5(71.4 \%)$ & \\
\hline \multicolumn{8}{|l|}{ Type of surgical treatment } \\
\hline Cervical procedure & $208(100.0 \%)$ & $102(100.0 \%)$ & $356(100.0 \%)$ & $41(100.0 \%)$ & $19(100.0 \%)$ & $7(100.0 \%)$ & - \\
\hline Repeated cervical procedure & $76(36.5 \%)$ & $20(19.6 \%)$ & $70(19.7 \%)$ & $4(9.8 \%)$ & $4(21.1 \%)$ & $0(0.0 \%)$ & $<0.001$ \\
\hline Sentinel lymph node biopsy & $55(26.4 \%)$ & $44(43.1 \%)$ & $171(48.0 \%)$ & $15(36.6 \%)$ & $9(47.4 \%)$ & $2(28.6 \%)$ & $<0.001$ \\
\hline Pelvic lymphadenectomy & $52(25.0 \%)$ & $81(79.4 \%)$ & $310(87.1 \%)$ & $32(78.0 \%)$ & $17(89.5 \%)$ & $5(71.4 \%)$ & $<0.001$ \\
\hline Paraaortic lymphadenectomy & $5(2.4 \%)$ & $1(1.0 \%)$ & $15(4.2 \%)$ & $0(0.0 \%)$ & $3(15.8 \%)$ & $0(0.0 \%)$ & 0.054 \\
\hline Cerclage placement & $22(10.6 \%)$ & $26(25.5 \%)$ & $106(29.8 \%)$ & $13(31.7 \%)$ & $3(15.8 \%)$ & $1(14.3 \%)$ & $<0.001$ \\
\hline \multicolumn{8}{|l|}{ Type of cervical procedure } \\
\hline Conization & $151(72.6 \%)$ & $54(52.9 \%)$ & $133(37.4 \%)$ & $5(12.2 \%)$ & $7(36.8 \%)$ & $2(28.6 \%)$ & $<0.001$ \\
\hline Laparoscopic radical trachelectomy & $1(0.5 \%)$ & $13(12.7 \%)$ & $30(8.4 \%)$ & $3(7.3 \%)$ & $0(0.0 \%)$ & $1(14.3 \%)$ & \\
\hline Radical abdominal trachelectomy & $18(8.7 \%)$ & $17(16.7 \%)$ & $93(26.1 \%)$ & $21(51.2 \%)$ & $5(26.3 \%)$ & $3(42.9 \%)$ & \\
\hline Radical vaginal trachelectomy & $18(8.7 \%)$ & $6(5.9 \%)$ & $67(18.8 \%)$ & $5(12.2 \%)$ & $3(15.8 \%)$ & $0(0.0 \%)$ & \\
\hline Robotic radical trachelectomy & $1(0.5 \%)$ & $5(4.9 \%)$ & $6(1.7 \%)$ & $2(4.9 \%)$ & $1(5.3 \%)$ & $0(0.0 \%)$ & \\
\hline Simple vaginal trachelectomy & $19(9.1 \%)$ & $7(6.9 \%)$ & $27(7.6 \%)$ & $5(12.2 \%)$ & $3(15.8 \%)$ & $1(14.3 \%)$ & \\
\hline \multicolumn{8}{|l|}{ Recurrence } \\
\hline No & $192(92.3 \%)$ & $96(94.1 \%)$ & $321(90.2 \%)$ & $33(80.5 \%)$ & $14(73.7 \%)$ & $7(100.0 \%)$ & 0.028 \\
\hline Yes & $16(7.7 \%)$ & $6(5.9 \%)$ & $35(9.8 \%)$ & $8(19.5 \%)$ & $5(26.3 \%)$ & $0(0.0 \%)$ & \\
\hline \multicolumn{8}{|l|}{ Type of recurrence } \\
\hline Pre-cancer & $8(3.8 \%)$ & $5(4.9 \%)$ & $9(2.5 \%)$ & $0(0.0 \%)$ & $0(0.0 \%)$ & $0(0.0 \%)$ & 0.623 \\
\hline Invasive cancer & $9(4.3 \%)$ & $2(2.0 \%)$ & $27(7.6 \%)$ & $8(19.5 \%)$ & $5(26.3 \%)$ & $0(0.0 \%)$ & $<0.001$ \\
\hline
\end{tabular}


Abstract 991 Table 2 Risk o recurrence (One-dimension logistic regression model)

\begin{tabular}{|c|c|c|}
\hline & OR $(95 \% \mathrm{Cl})$ & p-value \\
\hline \multicolumn{3}{|l|}{ Stage } \\
\hline T1a1 & Reference category & - \\
\hline T1a2 & $0.442(0.067-1.756)$ & 0.302 \\
\hline $\mathrm{T} 1 \mathrm{~b} 1 \leq 2 \mathrm{~cm}$ & $1.815(0.867-4.160)$ & 0.132 \\
\hline $\mathrm{T} 1 \mathrm{~b} 1>2 \mathrm{~cm}$ & $5.360(1.890-15.017)$ & 0.001 \\
\hline T1b2 & $7.897(2.188-26.316)$ & 0.001 \\
\hline $\mathrm{T} 2$ & - & - \\
\hline \multirow{2}{*}{\multicolumn{3}{|c|}{$\begin{array}{l}\text { Age at first diagnosis of cervical cancer } \\
\text { [years] }\end{array}$}} \\
\hline & & \\
\hline & $0.956(0.895-1.019)$ & 0.175 \\
\hline \multicolumn{3}{|l|}{ Histological type } \\
\hline Adeno & Reference category & - \\
\hline Adenosquamous & $1.228(0.270-4.087)$ & 0.759 \\
\hline Squamous & $0.841(0.440-1.696)$ & 0.611 \\
\hline Other & $19.038(2.917-$ & 0.002 \\
\hline & 154.774) & \\
\hline \multicolumn{3}{|l|}{ Largest size of the tumor [mm] } \\
\hline & $1.057(1.027-1.088)$ & $<0.001$ \\
\hline \multicolumn{3}{|l|}{ Type of cervical procedure } \\
\hline Conization & Reference category & - \\
\hline Laparoscopic radical trachelectomy & $0.652(0.102-2.315)$ & 0.571 \\
\hline Radical abdominal trachelectomy & $1.821(0.927-3.524)$ & 0.076 \\
\hline Radical vaginal trachelectomy & $0.968(0.348-2.318)$ & 0.945 \\
\hline Robotic radical trachelectomy & - & - \\
\hline Simple vaginal trachelectomy & $1.034(0.295-2.824)$ & 0.952 \\
\hline
\end{tabular}

was observed in tumours $\geq 2 \mathrm{~cm}$ in comparison to smaller tumours $(19.4 \%$ vs. $5.7 \%$; $<0.001)$. The most frequent locations of recurrence were cervix (53\%) and pelvic nodes (22\%). Median DFI for invasive recurrence reached 18 months.

Conclusion* Data from the real life practice showed that FST in cervical cancer patients is safe in patients with HPV related tumours smaller than $2 \mathrm{~cm}$. In such tumours conization represents sufficient procedure with satisfactory pregnancy outcomes. Surprisingly less than half of patients attempt to conceive after treatment.

\section{Miscellaneous}

\section{SGNTUC-019: PHASE 2 BASKET STUDY OF TUCATINIB AND TRASTUZUMAB IN SOLID TUMORS WITH HER2 ALTERATIONS: UTERINE AND CERVICAL CANCER COHORTS}

${ }^{1}$ BJ Monk, ${ }^{2} V$ Kang, ${ }^{2} \mathrm{~L}$ Walker, ${ }^{3} \mathrm{D}$ O'malley. ${ }^{1}$ Arizona Oncology (US Oncology Network), University of Arizona, Creighton University, Phoenix, AZ, USA; ${ }^{2}$ Seagen Inc., Bothell, WA, USA; ${ }^{3}$ Ohio State University, Columbus, OH, USA

\subsection{6/ijgc-2021-ESG0.249}

Introduction/Background* Tucatinib, a highly selective HER2directed tyrosine kinase inhibitor with minimal EGFR inhibition, is approved for use in combination with trastuzumab and capecitabine in patients with breast cancer who have received anti-HER2-based regimens in the metastatic setting. In xenograft models of HER2-overexpressed/amplified (HER2
+ ) and HER2-mutated tumors, dual targeting with tucatinib and trastuzumab showed superior activity to either agent alone.

The prognosis of locally-advanced unresectable or metastatic (LAUM) cervical and uterine cancer remains poor. HER2 amplification/overexpression and mutations occur in up to $21 \%$ and $80 \%$ of cervical and uterine cancers, respectively.

Methodology SGNTUC-019 (NCT04579380) is an open-label, international Phase 2 basket study evaluating tucatinib and trastuzumab in adult patients with LAUM HER2+ or HER2mutated solid tumors. Multiple disease- and HER2 alterationspecific cohorts are being enrolled, including HER2+ cervical and uterine cancer cohorts. Patients will receive tucatinib 300 mg orally twice daily and trastuzumab $8 \mathrm{mg} / \mathrm{kg}$ IV on Cycle 1 Day 1 and $6 \mathrm{mg} / \mathrm{kg}$ q21 days from Cycle 2 Day 1

HER2 + cervical and uterine cancer cohorts will enroll 12 patients each. If $\geq 2$ responses are observed in a cohort, it will be expanded to 30 patients. Patients with HER2-mutated cervical and uterine cancers will enroll in a cohort of 30 patients for all solid tumor types.

Eligible patients must have progressed on or after the last systemic therapy, with platinum-based therapy \pm bevacizumab required in patients with metastatic cervical cancer. Patients must have ECOG PS $\leq 1$, adequate organ function, and have not received HER2-directed therapy; patients with uterine serous carcinoma may have received trastuzumab. HER2 alterations can be demonstrated by HER2 overexpression/amplification in tumor tissue by prior IHC/ISH, or by HER2 amplification/mutation in a prior or on-study NGS assay of ctDNA or prior tissue NGS assay.

The primary endpoint is confirmed ORR per investigator. Disease control rate, duration of response, PFS, and OS are the secondary endpoints. Disease assessments per RECIST 1.1 will occur q6 weeks for 24 weeks, then q12 weeks. QoL will be evaluated q2 cycles using EQ-5D-5L.

Result(s)* Not applicable.

Conclusion* Enrollment in US began in Dec 2020; EU and Asia sites will be opened.

\section{SURGICAL AND MEDICAL TREATMENTS FOR UTERINE PECOMAS}

G Bogani*, A Gronchi, A Ditto, F Raspagliesi*. Fondazione IRCCS Istituto Nazionale dei Tumori, Milano, Italy

\subsection{6/ijgc-2021-ESG0.250}

Introduction/Background* Perivascular epitheliod cell tumors (PEComas) are rare mesenchymal neoplasms. Uterine PEComa is extremely rare and only limited evidence is still available. Methodology This is a single-center retrospective study. Charts of consecutive patients who had treatment (from 01/01/2010 to $12 / 31 / 2020$ ) for newly diagnosed uterine PEComas were retrieved. Five-year survival outcomes were assessed using Kaplan-Meier and Cox proportional hazard models.

Result(s)* Data of 23 patients with newly diagnosed PEComas were analyzed. Mean (SD) patients" age was 52 (14) years. Twenty-two patients had a surgical cytoreductive attempt. In one case surgery was not performed due to the presence of an extra-abdominal spread. Overall, seven (30\%) patients had disease outside the uterus at the time of surgery. Complete cytoreduction (no macroscopic residual tumor) was achieved in 19 patients. Complete cytoreduction was not completed in 